\title{
Sequences of embedded minimal disks whose curvatures blow up on a prescribed subset of a line
}

\author{
DAVID HOFFMAN AND BRIAN WhITE
}

For any prescribed closed subset of a line in Euclidean 3-space, we construct a sequence of minimal disks that are properly embedded in an open solid cylinder around the line and that have curvatures blowing up precisely at the points of the closed set.

\section{Introduction}

In this paper, we prove the following result about convergence of properly embedded minimal disks to a lamination:

\section{Theorem 0.1. Let}

$$
C=\mathbf{B}(0,1) \times(a, b) \subset \mathbf{R}^{3}
$$

be an open solid circular cylinder, possibly infinite, that is rotationally symmetric about the z-axis, $Z$. Let $K$ be a relatively closed subset of $Z \cap C$. Then there exists a sequence of minimal disks that are properly embedded in $C$ and that have the following properties:

(1) The curvatures of the disks are uniformly bounded on compact subsets of $C \backslash K$, and the curvatures blow up at each point of $K{ }^{1}$

(2) The minimal disks converge to a limit lamination of $C \backslash K$ consisting of the following leaves:

(i) For each $p \in K$, the horizontal punctured unit disk centered at $p$ :

$$
\mathcal{D}_{p}=(C \backslash Z) \cap\{z=z(p)\}
$$

\footnotetext{
${ }^{1}$ The curvatures of a sequence of minimal disks $D_{n}$ blow up at a point $p$ if there exists a sequence $\left\{p_{n} \in D_{n}\right\}$ with $p_{n} \rightarrow p$ such that the absolute value of the curvature of $D_{n}$ at $p_{n}$ tends to infinity as $n \rightarrow \infty$
} 
(ii) For each component $J$ of $(Z \backslash K) \cap C$, a leaf $\mathcal{M}_{J}$ that is properly embedded in the cylinder

$$
\mathbf{B}(0,1) \times z(J)
$$

The leaf $\mathcal{M}_{J}$ contains the segment $J$ and is therefore symmetric under rotation by $180^{\circ}$ around $Z$. Each of the two connected components of $\mathcal{M}_{J} \backslash J$ is an infinitely sheeted multigraph over the punctured unit disk, and $\partial z / \partial \theta>0$ everywhere on $\mathcal{M}_{J} \backslash J$.

(3) The lamination extends smoothly to a lamination of $C \backslash \partial K$, but it does not extend smoothly to any point in $C \cap \partial K$.

Here $z(J)$ denotes the image of $J$ under the coordinate map $z: \mathbf{R}^{3} \rightarrow \mathbf{R}$.

Note that according to Statement 2ii, each of the components of $\mathcal{M}_{J} \backslash J$ can be parametrized as

$$
(r, \theta) \in(0,1) \times \mathbf{R} \mapsto(r \cos \theta, r \sin \theta, f(r, \theta))
$$

Furthermore, if we let $(c, d)=z(J)$, then (by properness)

$$
\begin{array}{r}
\lim _{\theta \rightarrow-\infty} f(r, \theta)=c, \\
\lim _{\theta \rightarrow \infty} f(r, \theta)=d,
\end{array}
$$

the convergence being uniform away from $r=0$.

Theorem 0.1 is well known in case $K$ is the entire interval $Z \cap C$ : let the $n$th disk be the standard helicoid scaled by a factor of $1 / n$ and restricted to $C$. Theorem 0.1 was proved by Colding and Minicozzi [3] when $K$ consists of a single point, by Dean [6] when $K$ is an arbitrary finite set of points, and very recently by Kahn [9] when $K$ is an interval with exactly one endpoint in $C$. Our work was inspired by Kahn's result, although the methods are very different: Kahn, like Colding, Minicozzi and Dean used the Weierstrass representation, whereas our approach is variational. Kleene has given a different proof of Theorem 0.1 using the Weierstrass representation; see [10].

In the study of minimal varieties, the known examples of singularities have been rather tame. In particular, we believe that Theorem 0.1 provides the first examples of Cantor sets of singularities and of singular sets with noninteger Hausdorff dimension.

Our paper is organized as follows. In Section 1, we discuss what is known in general about curvature blow up in sequences of properly embedded minimal disks. We prove Theorem 0.1 in Section 2. The proof depends on results 
in Sections 3 and 4. In Section 3, we prove existence and uniqueness theorems for embedded minimal disks with certain rotationally symmetric boundaries. In Section 4, we use Rado's theorem to deduce curvature estimates for our examples.

\section{The general context}

Our paper gives examples of curvature blow up in sequences of properly embedded minimal disks. In this section, we describe what is known in general about such curvature blow up. (The results described in this section are not used in the rest of the paper.)

Suppose that for each $n$ we have a minimal disk $D_{n}$ that is properly embedded in an open subset $U_{n}$ of $\mathbf{R}^{3}$, where $U_{n} \subset U_{n+1}$ for each $n$. Let $U=\cup_{n} U_{n}$. By passing to a subsequence, we may suppose that there is a relatively closed subset $K$ of $U$ such that the curvatures of the $D_{n}$ blow up at all points of $K$ and such that the $D_{n}$ converge smoothly in $U \backslash K$ to a limit lamination $\mathcal{L}$ of $U \backslash K$. It is natural to ask how general the set $K$ can be, and to what extent the lamination and/or the leaves of the lamination can be smoothly extended to include points in $K$. In particular,

Q1. If $p$ is a point in the blow-up set $K$, must there be a leaf of the lamination that extends smoothly across $p$ ?

Q2. Must $K$ locally be contained in a nice (e.g., $C^{1}$ or perhaps $C^{1,1}$ ) curve?

Q3. Given an arbitrary closed subset $S$ of a nice curve in the open set $U$, is there an example for which which the blow-up set $K$ is precisely $S$ ?

(More precisely, Q2 is: must each point $p \in K$ have a neighborhood $W$ such that $K \cap W$ is, after a rotation, contained in the graph of a $C^{1}$ (or $C^{1,1}$ ) function from $\mathbf{R}$ to $\mathbf{R}^{2}$ ?)

According to Theorem 5.8 of [4], the answer to question Q1 is "yes." In particular, every point of $K$ has a neighborhood $W$ such that if $p \in K \cap W$, then there is a leaf $L_{p}$ of the limit lamination such that $L_{p}^{\prime}:=\left(L_{p} \cup\{p\}\right) \cap$ $W$ is a properly embedded minimal submanifold of $W$. Furthermore, if we choose $W$ small enough, then the $L_{p}^{\prime}$ are all graphs over a common plane (with uniformly small slopes). Note that for each $p \in K \cap W$, there is only one such $L_{p}^{\prime}$ by the strong maximum principle.

The answer to Q2 is also "yes," at least in the $C^{1}$ case. That the answer is "yes" for Lipschitz curves is implicit in the work of Colding and Minicozzi; see, for example, [5, Section I.1] and [5, Theorem 0.1] for a very similar result. Assuming the results of Colding and Minicozzi and an improvement by Meeks [11], [15] proves that the answer to Q2 is "yes" in the $C^{1}$ case. 
Colding and Minicozzi also proved that if $U=\mathbf{R}^{3}$ (the so-called "global case") and if $K$ is nonempty, then (after a rotation) $K$ is the graph of a Lipschitz function $z \in \mathbf{R} \mapsto(x(z), y(z))$ and the lamination is the foliation by horizontal planes punctured at the points of $K$ [5, Theorem 0.1$]$. In particular, the lamination extends smoothly to all of $\mathbf{R}^{3}$. By [11] (described below), the curve is in fact a straight line that is perpendicular to the planes.

In the local case $U \neq \mathbf{R}^{3}$, the behavior can be very different, as Theorem 0.1 indicates. Meeks proved that if the lamination extends smoothly to a foliation of $U$ and if $K$ is a Lipschitz curve that intersects the leaves transversely, then $K$ is a $C^{1,1}$ curve and it intersects the leaves orthogonally [11]. Meeks and Weber [12] constructed an example for which $U=\mathbf{R}^{3} \backslash Z$, the blow-up set $K$ is a horizontal circle centered at a point in $Z$, and the limit lamination consists of the vertical half-planes with $Z$ as edge, punctured by $K$. They go on to prove that, given any $C^{1,1}$ curve, there is an example in which the blow-up set $K$ is that curve, $U$ is tubular neighborhood of $K$, and the lamination is a foliation of $U$ by planar punctured disks orthogonal to $K$. The examples of Meeks and Weber and the examples in this paper suggest that the answer to Q3 may be "yes."

\section{The proof of Theorem 0.1}

Proof. It suffices to prove the theorem for the unbounded cylinder $C=$ $\mathbf{B}(0,1) \times \mathbf{R}$ : the case of bounded cylinders follows by restriction.

For each connected component $J$ of $Z \backslash K$, choose a smooth embedded curve $S_{J}$ in $\partial \mathbf{B}(0,1) \times J$ such that (see figure 1)

(1) The projection $(x, y, z) \mapsto(0,0, z)$ induces a diffeomorphism from $S_{J}$ to $J$.

(2) The derivative $d \theta / d z$ is strictly positive at each point of $S_{J}$. (Here $\theta$ is the angle of the cylindrical coordinate of a point in $\mathbf{R}^{3} \backslash Z$. Of course, $\theta$ is defined only up to an integer multiple of $2 \pi$, but $d \theta / d z$ is well defined.)

(3) The curve $S_{J}$ winds around the cylinder infinitely many times as $z \rightarrow c$ and as $z \rightarrow d$, where $c$ and $d$ are the infimum and supremum, respectively, of $z$ on $J$. In other words, $\lim _{z \rightarrow c} \theta(z)=-\infty$ and $\lim _{z \rightarrow d}$ $\theta(z)=\infty$. 




Figure 1: The curve $S_{J}$ on $\partial \mathbf{B}(0,1) \times \mathbf{R}$, as described in conditions (1), (2) and (3). Here, $J$ is a bounded open interval of the axis $Z$, the endpoints of which are in the closed set $K$. The limit set of $S_{J}$ consists of the two horizontal circles on $\partial \mathbf{B}(0,1) \times \mathbf{R}$ at the heights of the endpoints of $J$.

Now choose numbers $a_{n} \rightarrow-\infty$ and $b_{n} \rightarrow \infty$, and choose choose smooth curves $\gamma_{n}$ in $\partial \mathbf{B}(0,1) \times\left[a_{n}, b_{n}\right]$ such that

(i) The curve $\gamma_{n}$ can be smoothly parametrized by $z \in\left[a_{n}, b_{n}\right]$, (that is, $(x, y, z) \mapsto z$ induces a diffeomorphism from $\gamma_{n}$ to $\left.\left[a_{n}, b_{n}\right]\right)$.

(ii) The derivative $d \theta / d z$ is strictly positive at each point of $\gamma_{n}$.

(iii) The $\gamma_{n}$ converge smoothly to the lamination of $\partial C$ consisting of the $S_{J}$ 's together with the horizontal circles of radius 1 centered at points of $K$.

Let $\Gamma_{n}$ be the simple closed curve consisting of $\gamma_{n}$ and $Z \cap\left\{a_{n} \leq z \leq\right.$ $\left.b_{n}\right\}$, together with two radial segments at heights in $z=a_{n}$ and $z=b_{n}$. By Theorem 3.2 in Section $3, \Gamma_{n}$ bounds a unique embedded minimal disk $D_{n}$.

Extend $D_{n}$ by repeated Schwartz reflection in the the top and bottom edges to get an infinite minimal strip $\tilde{D}_{n}$. The boundary of $\tilde{D}_{n}$ has two components: the axis $Z$, and the helix-like curve $\tilde{\gamma}_{n}$ in $\partial C$ obtained from $\gamma_{n}$ by iterated reflection.

To ensure that the boundary curve $\tilde{\gamma}_{n}$ is smooth, we need to impose one additional condition on the choice of $\gamma_{n}$ :

(iv) The even-order derivatives $d^{k} \theta / d z^{k}(k=2,4, \ldots)$ vanish at the endpoints of $\gamma_{n}$. 
(If this condition were not satisfied, then the curve $\tilde{\gamma}_{n}$ would not be smooth at the endpoints of $\gamma_{n}$.)

By Theorem 3.2, $\tilde{D}_{n}$ and its rotated images $R_{\theta} \tilde{D}_{n}$ foliate $C \backslash Z$. Thus if $D_{n}^{*}=\tilde{D}_{n} \cup \rho_{Z} \tilde{D}_{n} \cup Z$ is the minimal surface obtained from $\tilde{D}_{n}$ by Schwartz reflection in $Z$, then $D_{n}^{*}$ is embedded. Here $\rho_{Z}=R_{\pi}$ denotes rotation by $\pi$ about $Z$.

The disks $D_{n}^{*}$ are the disks whose existence is asserted in the statement of the theorem. We now prove that they have the properties asserted by the theorem.

By Theorem 3.2 applied to $D_{n}$, each of the two connected components of $D_{n}^{*} \backslash Z$ is a multigraph over $\mathbf{B}(0,1) \backslash\{0\}$. (Of course those components are $\tilde{D}_{n}$ and $\rho_{Z} \tilde{D}_{n}$.) Thus if $H$ is an open half-space bounded by a plane containing $Z$, then $H \cap D_{n}^{*}$ is a union of graphs over a half-disk. Standard estimates for solutions of the minimal surface equation then imply that the principal curvatures of the closures of the $D_{n}^{*}$ are uniformly bounded on compact subsets of $H$. Thus after passing to a subsequence, those graphs will converge smoothly (away from $\partial H$ ) to a collection $\mathcal{G}_{H}$ of minimal graphs over a half-disk. Note that if $G$ is such a graph, then $(\partial G) \cap H$ must be one of the following:

(H1) A horizontal semicircle at height $z(p)$ for some $p \in K$, or

(H2) one of the connected components of $S_{J} \cap H$ or one of the connected components of $\left(\rho_{Z} S_{J}\right) \cap H$, where $J$ is a connected component of $Z \backslash K$.

Furthermore,

(H3) If $\Gamma$ is a connected component of $S_{J} \cap H$ or of $\left(\rho_{Z} S_{J}\right) \cap H$, then there is exactly one graph $G$ in $\mathcal{G}_{H}$ whose boundary (in $H$ ) is $\Gamma$.

Since $H$ is arbitrary, this means that (after passing to a subsequence) the $D_{n}^{*} \backslash Z$ will converge smoothly on compact subsets of $\mathbf{R}^{3} \backslash Z$ to a lamination $\mathcal{L}$ of $C \backslash Z$ consisting of a union of multigraphs. Because each leaf is embedded, it must either have a single sheet (and thus be a graph) or else have infinitely many sheets.

Let $J$ be a component of $Z \backslash K$. By Theorem 4.1, principal curvatures of the $D_{n}^{*}$ are uniformly bounded ${ }^{2}$ on compact subsets of $\mathbf{B}(0,1) \times z(J)$. Since the principal curvatures are also uniformly bounded on compact subsets

\footnotetext{
${ }^{2}$ We could apply standard curvature estimates instead of Theorem 4.1 if we had uniform area bounds in little balls centered along $Z$, but proving the necessary area bounds does not seem to be any easier than proving Theorem 4.1.
} 
of $\mathbf{R}^{3} \backslash Z$, this means that the curvatures are in fact uniformly bounded on compact subsets of $\mathbf{R}^{3} \backslash K$. Thus (perhaps after passing to a further subsequence) the $D_{n}^{*}$ converge smoothly on compact subsets of $C \backslash K$ to a lamination $\mathcal{L}^{\prime}$ of that region. Of course, $\mathcal{L}$ is the restriction of $\mathcal{L}^{\prime}$ to the gutted cylinder $C \backslash Z$.

Claim 2.1. The horizontal circle $\mathcal{C}_{p}$ of radius 1 centered at a point $p \in$ $K$ bounds a unique leaf of $\mathcal{D}_{p} \in \mathcal{L}^{\prime}$. That leaf is the planar punctured disk bounded by $\mathcal{C}_{p}$.

Proof of Claim 2.1. As above, let $H$ be an open half-space of $\mathbf{R}^{3}$ with $Z \subset$ $\partial H$. Then $\mathcal{L}^{\prime} \cap H$ is a union of minimal graphs over a half-disk. Consider the set $Q$ of those graphs that contain $\mathcal{C}_{p} \cap H$ in their boundaries. That set is compact, so there is an uppermost graph $G_{H}$ in $Q$. Note that the union of the $G_{H}$ as $H$ varies (by rotating it around $Z$ ) forms a single smooth minimal graph $G$ over the punctured disk $\mathbf{B}(0,1) \backslash\{0\}$. That graph satisfies the minimal surface equation. As a minimal surface in $\mathbf{R}^{3}$, the boundary of $G$ is the circle $\mathcal{C}_{p}$ together with some or all of $Z$. Since a solution to the minimal surface equation cannot have an isolated interior singularity, the graph extends to a regular minimal surface over $\mathbf{B}(0,1)$. (see Theorem 10.2 of [14]. The result is originally due to Bers [1], but the proof in [14] using catenoidal barriers is due to Finn [7].) Since the boundary values define a planar circle, the graph must be a flat disk, so $G$ is a flat planar disk. Recall that $G$ is the uppermost leaf of $\mathcal{L}$ that contains $\mathcal{C}_{p}$. By the same argument, it is also the lowermost leaf of $\mathcal{L}$ containing $\mathcal{C}_{p}$. Thus it is the unique leaf in $\mathcal{L}$ containing $\mathcal{C}_{p}$.

Claim 2.2. If $p \in K$, then there is a sequence $p_{n} \in D_{n}^{*}$ converging to $p$ such that the norm of the second fundamental form of $D_{n}^{*}$ at $p_{n}$ tends to infinity.

Proof of Claim 2.2. Suppose not. Then there is a ball $\mathbf{B} \subset \mathbf{R}^{3}$ centered at $p$ and a subsequence of the $D_{n}^{*}$ (which we may take to be the original sequence) such that the curvatures of the $D_{n}^{*}$ are uniformly bounded in B. It follows that the lamination $\mathcal{L}^{\prime}$ extends smoothly to a lamination of $\mathbf{B}$ and that the convergence of $D_{n}^{*} \cap \mathbf{B}$ to the lamination of $\mathbf{B}$ is smooth. By Claim 1 , the leaf containing $p$ is a horizontal disk. But each $D_{n}^{*}$ contains the axis $Z$, and therefore has a vertical tangent plane at $p$. Hence the convergence cannot be smooth. This contradiction proves the claim. 
We have now completely established Statement 1 of the Theorem 0.1 , and we have established that there is a limit lamination of $C \backslash K$. We also know that for each $p \in K$, the punctured disk $\mathcal{D}_{p}$ is a leaf of this lamination. Thus we have proved Statement $2 \mathrm{i}$.

To prove Statement 2ii, let $J=\{p \in Z: c<z(p)<d\}$ be one of the components of $Z \backslash K$. We now analyze the leaves of the foliation that lie between the punctured disks at heights $c$ and $d$. Let $\mathcal{M}_{J}^{+}$and $\mathcal{M}_{J}^{-}$be the leaves of $\mathcal{L}$ that contain $S_{J}$ and $\rho_{Z}\left(S_{J}\right)$, respectively. Note that by $(\mathrm{H} 1)$ and (H2), these are both infinite covers of $\mathbf{B}(0,1) \backslash\{0\}$. By (H3), there are no other leaves of $\mathcal{L}$ in the region $\{c<z<d\}$.

Note that $J \subset D_{n}^{*}$ for every $n$, so $J$ is contained in one of the leaves $\mathcal{M}_{J}$ of the lamination $\mathcal{L}^{\prime}$. Now $\mathcal{M}_{J}$ is simply connected since each $D_{n}^{*}$ is simply connected, so $\mathcal{M}_{J} \backslash Z$ must contain two components. These components are leaves of $\mathcal{L}$, so they must be $\mathcal{M}_{J}^{+}$and $\mathcal{M}_{J}^{-}$. Thus $\mathcal{M}_{J}=J \cup \mathcal{M}_{J}^{+} \cup \mathcal{M}_{J}^{-}$is the unique leaf of $\mathcal{L}^{\prime}$ in the slab $\{c<z<d\}$.

Let $H$ be an open half-space of $\mathbf{R}^{3}$ bounded by a plane containing $Z$. Note that the components of $\mathcal{M}_{J} \cap H$ form a countable discrete set corresponding to the countable discrete set of components of $\left(S_{J} \cup \rho_{Z} S_{J}\right) \cap H$. Thus $\mathcal{M}_{J}$ is not a limit leaf of the foliation $\mathcal{L}^{\prime}$.

It follows that $\mathcal{M}_{J}$ is properly embedded in $\Omega:=\mathbf{B}(0,1) \times(c, d)$. For if not, $\mathcal{L}^{\prime}$ would have a limit leaf in $\Omega$. But the only leaf in $\Omega$ is $\mathcal{M}_{J}$ itself, which, as we have just seen, is not a limit leaf. This proves properness.

Next we show that $\partial z / \partial \theta>0$ on $\mathcal{M}_{J} \backslash J$. (The partial derivative makes sense because $\mathcal{M}_{J} \backslash J$ is locally a graph.) Since the two components of $\mathcal{M}_{J} \backslash J$ are related by the $\rho_{Z}$ symmetry, it suffices to show that $\partial z / \partial \theta>0$ on the component $M=\mathcal{M}_{J}^{+}$. Let $\nu$ be the unit normal vectorfield on $M$ given by

$$
\nu:=\frac{(\nabla z,-1)}{W}
$$

where $\nabla z=\left(\frac{\partial z}{\partial x}, \frac{\partial z}{\partial y}\right)$ and $W=|(\nabla z,-1)|$. Note that the Killing field

$$
\frac{\partial}{\partial \theta}:=\left(\frac{\partial x}{\partial \theta}, \frac{\partial y}{\partial \theta}, 0\right)=(-y, x, 0)
$$

restricted to $M$ is the initial velocity vectorfield of the one-parameter family $\theta \mapsto R_{\theta} M$ of minimal surfaces. Thus the function

$$
\begin{aligned}
& u: M \rightarrow \mathbf{R} \\
& u=\nu \cdot \frac{\partial}{\partial \theta}=W^{-1}\left(\frac{\partial z}{\partial x} \frac{\partial x}{\partial \theta}+\frac{\partial z}{\partial y} \frac{\partial y}{\partial \theta}\right)=W^{-1} \frac{\partial z}{\partial \theta}
\end{aligned}
$$


satisfies the Jacobi field equation

$$
\Delta u+|A|^{2} u=0
$$

where $A$ is the second fundamental form of $M$. (See [2, Lemma 1].)

Now $\partial z / \partial \theta \geq 0$ on the disks $D_{n}$ (by Theorem 3.2), so $\partial z / \partial \theta \geq 0$ on $M$. Thus $u$ is non-negative, so from the Jacobi field equation, we see that $u$ is superharmonic. Hence by the maximum principle, $u$ is either everywhere 0 or everywhere strictly positive. The same is true of $\partial z / \partial \theta=W u$. Since $\partial z / \partial \theta>0$ along the outer boundary curve $S_{J}$ of $M$ (by choice of $S_{J}$ ), it must be positive everywhere on $M$.

It remains to prove Statement 3 of the theorem. By Claim 2.1, the punctured disks $\mathcal{D}_{p}$ with $p \in K$ are leaves of $\mathcal{L}^{\prime}$. Each such leaf can be extended smoothly to include the puncture. This extension of $\mathcal{L}^{\prime}$ is smooth near any point in the interior of $K$.

However, if $p \in \partial K$, then $p$ is simultaneously the limit of points in $\mathcal{D}_{p}$ and the limit of points in $Z \backslash K$. At the former points, the tangent planes to $\mathcal{L}^{\prime}$ are horizontal, whereas at the latter points the tangent planes are vertical. Thus the lamination $\mathcal{L}^{\prime}$ cannot extend smoothly to any points in $\partial K$.

\section{Existence and uniqueness of embedded minimal disks with rotationally symmetric boundaries}

Theorem 3.1. Let $W$ be a nonempty, bounded, convex open subset of $\mathbf{R}^{3}$ that is rotationally symmetric about $Z$ and let $I=W \cap Z$. Let $R_{\theta}$ be rotation about $Z$ through angle $\theta$. Suppose $\Gamma$ is a piecewise smooth simple closed curve such that $\Gamma$ contains $I$, such that $\Gamma \backslash I$ is contained in $\partial W$, and such that the curves

$$
R_{\theta}(\Gamma \backslash \bar{I}), \quad 0 \leq \theta<2 \pi,
$$

foliate $(\partial W) \backslash Z$. Then $\Gamma$ bounds a unique embedded minimal disk D. Furthermore, the disks

$$
R_{\theta} D, \quad 0 \leq \theta<2 \pi
$$

foliate $W \backslash I$.

Proof. Let

$$
\gamma=\{(r, z): r \geq 0 \text { and }(r, 0, z) \in \partial W\}
$$


Let $\mathcal{F}$ be the set of piecewise smooth functions $f: \gamma \rightarrow \mathbf{R}$ for which the curve

$$
\Gamma_{f}=\{(r \cos f(r, z), r \sin f(r, z), z):(r, z) \in \gamma\} \cup I
$$

bounds an embedded minimal disk $D_{f}$ whose rotated images $R_{\theta} D_{f}$ foliate $W \backslash Z$.

Note that if $f$ is constant, then $\Gamma_{f}$ is a planar curve that bounds a planar disk whose rotated images foliate $W \backslash I$. Thus $\mathcal{F}$ contains all the constant functions.

Claim 3.1. Suppose that $f \in \mathcal{F}$ and that $g: \gamma \rightarrow \mathbf{R}$ is a piecewise smooth function such that

$$
\sup \|f-g\|<\pi / 4 \text {. }
$$

Then $g$ must also belong to $\mathcal{F}$.

Since $\mathcal{F}$ is nonempty, the claim implies that $\mathcal{F}$ contains every piecewise smooth function. Thus once we have proved the claim, we will have proved the existence part of the theorem, because every $\Gamma$ satisfying the hypotheses of the theorem can be written in the form (3.1).

Proof of Claim 3.1. Let

$$
\Omega=\bigcup_{-\pi / 4 \leq \theta \leq \pi / 4} R_{\theta} D_{f}
$$

Note that $\Omega$ is mean convex and simply connected and that $\Gamma_{g}$ is contained in $\partial \Omega$. Hence, $\Gamma_{g}$ bounds a least-area disk $D_{g}$ in $\Omega$.

Suppose that the $R_{\theta} D_{g}$ do not foliate $W \backslash Z$. Then $D_{g}$ and $R_{\theta} D_{g}$ intersect each other for some $\theta \in(0, \pi)$. Any such $\theta$ must in fact be less than $\pi / 2$ because

$$
\begin{gathered}
D_{g} \subset \Omega, \\
R_{\theta} D_{g} \subset R_{\theta} \Omega,
\end{gathered}
$$

and because $\Omega$ and $R_{\theta} \Omega$ are disjoint for $\pi / 2 \leq \theta \leq \pi$. Thus if $\alpha$ is the supremum of $\theta \in(0, \pi)$ for which $D_{g}$ and $R_{\theta} D_{g}$ intersect each other, then $0<\alpha \leq \pi / 2$. The boundary curves $\Gamma_{g}$ and $R_{\alpha} \Gamma_{g}$ intersect only on $\bar{I}$, so $D_{g}$ and $R_{\alpha} D_{g}$ must be tangent at some point in $D_{g} \cup \bar{I}$. At each point of $\bar{I}$, the disks $D_{g}$ and $R_{\alpha} D_{g}$ make an angle of $\alpha \neq 0$ with each other. So the point of tangency must lie in $D_{g}$. This contradicts the maximum principle. Hence 
the rotated images of $D_{g}$ foliate $W \backslash Z$. In particular, $D_{g}$ is embedded. This completes the proof of the claim.

We have proved the existence of a disk $D$ with boundary $\Gamma \cup \bar{I}$ such that the rotated images of $D$ foliate $W \backslash I$. It remains only to prove uniqueness. (In this paper, we never actually use the uniqueness.) Let $\Sigma$ be any embedded minimal disk with boundary $\Gamma$. Since $\Sigma$ is embedded, it has no boundary branch points, so that $\Sigma \cup I$ is a smooth manifold with boundary.

Since the disks $R_{\theta} D$ foliate $W \backslash I$, there is a unique continuous function

$$
\omega: \bar{\Sigma} \rightarrow \mathbf{R}
$$

such that

(1) $\omega=0$ on $\Gamma$,

(2) $p \in R_{\omega(p)} D$ for $p \in \Sigma$, and

(3) for $p \in I, R_{\omega(p)} D$ and $\Sigma$ have the same tangent half-plane.

(If $\Sigma$ were not simply connected, $\omega$ might only be well-defined up to multiples of $2 \pi$. That is, $\omega$ would take values in $\mathbf{R} / 2 \pi \mathbf{Z}$. But since $\Sigma$ is simply connected, we can lift $\omega$ to the universal cover $\mathbf{R}$ of $\mathbf{R} / 2 \pi \mathbf{Z}$.)

By the maximum principle and the boundary maximum principle (applied to points in $I$ ), the maximum value of $\omega$ must be attained on $\Gamma$. Thus the maximum value of $\omega$ is 0 . Similarly, the minimum value is 0 . Thus $\omega$ is identically 0 , so $\Sigma=D$.

Theorem 3.2. Let

$$
\phi:[a, b] \rightarrow \mathbf{R}
$$

be a smooth, strictly increasing function. Let $\Gamma$ be the closed curve consisting of

$$
(\cos \phi(z), \sin \phi(z), z), \quad a \leq z \leq b
$$

together with the segment $I=Z \cap\{a<z<b\}$ and two horizontal segments in the planes $z=a$ and $z=b$.

Then $\Gamma$ bounds a unique embedded minimal disk $D$. The rotated images $R_{\theta} D$ foliate $C \backslash Z$, where $C$ is the cylinder $\mathbf{B}(0,1) \times(a, b)$. The disk $D$ can 
be parametrized as

$$
(r, \theta) \mapsto(r \cos \theta, r \sin \theta, f(r, \theta))
$$

for some function

$$
f:[0,1] \times[\alpha, \beta] \rightarrow \mathbf{R},
$$

where $\alpha=\phi(a)$ and $\beta=\phi(b)$. That is, $D$ is a multigraph over $\mathbf{B}(0,1) \backslash\{0\}$. Furthermore, $f(r, \theta)$ is a strictly increasing function of $\theta$ for each $r$. In particular, $\partial f / \partial \theta$ is everywhere nonnegative.

Remark 3.1. In fact, $\partial f / \partial \theta$ is everywhere strictly positive. The proof is almost identical to the proof that $\partial z / \partial \theta>0$ in Theorem $0.1(2 \mathrm{ii})$.

Proof. Observe that $\Gamma \backslash I$ lies on the boundary of the solid cylinder $C=$ $\mathbf{B}(0,1) \times(a, b)$, and that the rotated images of $\Gamma \backslash \bar{I}$ foliate $\partial C \backslash \bar{I}$. Thus by Theorem 3.1, $\Gamma$ bounds a unique embedded minimal disk $D$, and the rotated images $R_{\theta} D$ foliate $C \backslash I$.

Note that since $D$ is simply connected, there is a continuous function $\theta: \bar{D} \backslash I \rightarrow \mathbf{R}$ such that for $p=(x, y, z) \in \bar{D} \backslash I$,

$$
(x, y)=\sqrt{x^{2}+y^{2}}(\cos \theta(p), \sin \theta(p)) .
$$

Note also that we can choose $\theta$ so that for $(x, y, z) \in \Gamma \backslash I$,

$$
\theta(x, y, z)=\phi(z)
$$

In particular, $\theta \equiv \alpha$ on $\Gamma \cap\{z=a\}$ and $\theta \equiv \beta$ on $\Gamma \cap\{z=b\}$, where $\alpha=$ $\phi(a)$ and $\beta=\phi(b)$.

Since $D \cup I$ is a smooth manifold with boundary, the angle function $\theta$ extends smoothly to $I$. (If this is not clear, consider a point $(0,0, c) \in I$. Then $(D \cup I) \cap\{z=c\}$, i.e., $(D \cap\{z=c\}) \cup\{(0,0, c)\}$ is a smooth curve. Thus it has a well-defined tangent half-line at the endpoint $(0,0, c)$, which implies that $\lim _{(x, y, c) \in D \rightarrow(0,0, c)} \theta(x, y, c)$ exists. Define $\theta(0,0, c)$ to be that limit. The smoothness of $D \cup I$ implies that this extension of $\theta$ to $D \cup I$ is smooth.)

By the maximum principle, $\theta$ cannot attain its maximum or its minimum at any interior point of $D$. By the boundary maximum principle, $\theta$ cannot attain its maximum or minimum at any point of $I$. Thus the maximum and minimum are attained on $\Gamma \backslash I$, so the minimum value is $\alpha$ and the maximum value is $\beta$. 
To show that $D$ is a multigraph, it suffices to show that the map

$$
p \in \bar{D} \mapsto(r(p), \theta(p)) \in[0,1] \times[\alpha, \beta]
$$

is one-to-one. (It is onto by elementary topology.)

Let

$$
S=\{(p, q) \in \bar{D} \times \bar{D}: r(p)=r(q) \text { and } \theta(p)=\theta(q)\}
$$

By compactness, the function

$$
(p, q) \in S \mapsto z(q)-z(p)
$$

attains its maximum value $h=z\left(q_{0}\right)-z\left(p_{0}\right)$ at some $\left(p_{0}, q_{0}\right) \in S$. To show that the map (3.2) is one-to-one, it suffices to show that $h=0$. To see that $h=0$, let $r_{0}:=r\left(p_{0}\right)=r\left(q_{0}\right)$ and $\theta_{0}:=\theta\left(p_{0}\right)=\theta\left(q_{0}\right)$.

If $r_{0}=1$ or if $\theta_{0}$ is $\alpha$ or $\beta$, then $p_{0}$ and $q_{0}$ are both in $\Gamma \backslash I$ by the maximum principle. But $p \mapsto(r(p), \theta(p))$ is one-to-one on $\Gamma \backslash I$ by choice of $\Gamma$, so in this case $h=0$ and we are done.

Thus we may suppose that $r_{0}<1$ and that $\alpha<\theta_{0}<\beta$. Now the minimal disks $\bar{D}$ and $\bar{D}+(0,0, h)$ are tangent at the point $q_{0}$, but in neighborhood of that point $\bar{D}$ lies on one side of $\bar{D}+(0,0, h)$. Thus by the strong maximum principle (if $r_{0}>0$ ) or by the strong boundary maximum principle (if $r_{0}=$ $0)$, the two disks coincide, which implies that $h=0$.

It remains to show that $f(r, \theta)$ is a strictly increasing function of $\theta$ for each $r$. Since the disks $R_{\alpha} D$ foliate $C \backslash Z$, it follows (for each fixed $r$ ) that the graphs of the curves

$$
\mathcal{C}_{\alpha}: \theta \mapsto f(r, \theta-\alpha)
$$

foliate the strip $\mathbf{R} \times(a, b)$. Thus the function $\theta \mapsto f(r, \theta)$ must be strictly monotonic. Since it is strictly increasing for $r=1$, it must be strictly increasing for all $r$.

\section{Curvature estimates via Rado's Theorem}

Theorem 4.1. Let $D \subset \mathbf{R}^{3}$ be a minimal disk contained in a vertical solid cylinder $C=\mathbf{B} \times \mathbf{R}$ of radius $R$, and let $\Gamma=\partial D$ be its boundary curve. Suppose that $\Gamma \cap\{a<z<b\}$ consists of two components, each of which is $a C^{1}$ curve whose tangent line has slope $\geq \epsilon>0$ in absolute value at every point. 
Let

$$
D_{\delta}=D \cap\{a+\delta<z<b-\delta\}
$$

where $\delta>0$. Then

(1) $D_{\delta}$ has no branch points.

(2) The slope of the tangent plane at each point of $D_{\delta}$ is greater than or equal to

$$
\min \left\{\epsilon, \frac{\delta}{2 R}\right\} \text {. }
$$

(3) For $p \in D_{\delta}$, the norm of the second fundamental form of $D_{\delta}$ at $p$ is bounded by

$$
\frac{C}{\operatorname{dist}\left(p, \partial D_{\delta}\right)}
$$

where $C$ is constant depending only on $\epsilon$ and $\delta / R$.

The proof of Theorem 4.1 uses the following theorem of Rado:

Theorem 4.2 (Rado). If the boundary of a minimal disk in $\mathbf{R}^{3}$ intersects a plane in fewer than 4 points, then $D$ has no branch points in the plane, and $D$ intersects the plane transversely.

See, for example,[14, Lemma 7.5] or [8, p. 272] for a proof.

Proof of Theorem 4.1. For $a<t<b$, the set $\Gamma \cap\{z=t\}$ contains exactly two points. Thus by Rado's Theorem, the surface $D \cap\{a<z<b\}$ has no branch points and no horizontal tangent planes.

Now let $p \in D_{\delta}$ and let $P$ be a plane through $p$ whose slope is less than $\epsilon$ and less than $\delta /(2 R)$. Since the slope is less than $\delta /(2 R)$, the plane $P$ does not intersect $\mathbf{B} \times(-\infty, a]$ or $\mathbf{B} \times[b, \infty)$. Thus

$$
P \cap \Gamma=P \cap(\Gamma \cap\{a<z<b\})
$$

Since the slope of $P$ is less than $\epsilon$, the plane $P$ intersects each of the the two components of $\Gamma \cap\{a<z<b\}$ in at most one point. Thus $P$ intersects $\Gamma$ in at most two points, so $P$ is not tangent to $D$ at $p$ by Rado's Theorem. This proves Assertion 2. 
Assertion 3 follows from Assertion 2 because Assertion 3 holds for any minimal surface $D_{\delta}$ whose image under the Gauss map omits a nonempty open subset of the unit sphere, the constant $C$ depending only on the size of that open set. This curvature estimate was proved by Osserman [13, Theorem 1], who even obtained the optimal constant. Alternatively, the estimate without the optimal constant follows by a standard blow-up argument from the fact (also due to Osserman [14, Theorem 8.1]) that the image of the Gauss map of a complete, nonflat minimal surface in $\mathbf{R}^{3}$ must be dense in the unit 2-sphere.

\section{Acknowledgments}

The research of B.W. was supported by the NSF under grant DMS-0104049.

\section{References}

[1] L. Bers, Isolated singularities of minimal surfaces, Ann. Math. 53(2) (1951), 364-386. MR0043335 (13,244c).

[2] J. Choe, Index, vision number and stability of complete minimal surfaces, Arch. Rational Mech. Anal. 109(3) (1990), 195-212. MR1025170 (91b:53007).

[3] T.H. Colding and W.P. Minicozzi II, Embedded minimal disks: proper versus nonproper - global versus local, Trans. Amer. Math. Soc. 356(1) (2004), 283-289 (electronic). MR2020033 (2004k:53011).

[4] - The space of embedded minimal surfaces of fixed genus in a 3-manifold. II. Multi-valued graphs in disks, Ann. Math. (2) 160(1) (2004), 69-92, DOI 10.4007/annals.2004.160.69. MR2119718 (2006a:53005).

[5] - The space of embedded minimal surfaces of fixed genus in a 3-manifold. IV. Locally simply connected, Ann. Math. (2) 160(2) (2004), 573-615. MR2123933 (2006e:53013).

[6] B. Dean, Embedded minimal disks with prescribed curvature blowup, Proc. Amer. Math. Soc. 134(4) (2006), 1197-1204 (electronic). MR2196057 (2007d:53009).

[7] R. Finn, Remarks relevant to minimal surfaces, and to surfaces of prescribed mean curvature, J. Analyse Math. 14 (1965), 139-160. MR0188909 (32 \#6337). 
[8] U. Dierkes, S. Hildebrandt, A. Küster and O. Wohlrab, Minimal surfaces. I, Grundlehren der Mathematischen Wissenschaften [Fundamental principles of mathematical sciences], 295, Springer-Verlag, Berlin, 1992. Boundary value problems. MR1215267 (94c:49001a).

[9] S. Kahn, A minimal lamination of the unit ball with singularities along a line segment, Illinois J. Math. (2008), to appear, available at arXiv:0902 .3641v2 [math.DG].

[10] S.J. Kleene, A minimal lamination with Cantor set-like singularities (2009), available at arXiv:0910.0199 [math.DG].

[11] W.H. Meeks III, Regularity of the singular set in the ColdingMinicozzi lamination theorem, Duke Math. J. 123(2) (2004), 329-334. MR2066941 (2005d:53014).

[12] W.H. Meeks III and Matthias Weber, Bending the helicoid, Math. Ann. 339(4) (2007), 783-798. MR2341900 (2008k:53020).

[13] R. Osserman, On the Gauss curvature of minimal surfaces, Trans. Amer. Math. Soc. 96 (1960), 115-128. MR0121723 (22 \#12457).

[14] - A survey of minimal surfaces, 2nd ed., Dover Publications Inc., New York, 1986. MR852409 (87j:53012).

[15] B. White, Curvatures of embedded minimal disks blow up on subsets of $C^{1}$ curves (2011), (preprint available at arXiv:0905.0851 [math.DG]).

Department of Mathematics

STANFORD UNIVERSITY

STANFORD

CA 94305

USA

E-mail address: hoffman@math.stanford.edu

E-mail address: white@math.stanford.edu

ReCeived June 3, 2010 